ESAIM: PROCEEDINGS AND SURVEYS, January 2015, Vol. 48, p. 385-399

N. Champagnat, T. Lelièvre, A. Nouy, Editors

\title{
UNCERTAINTY RELATED TO HIGH RESOLUTION TOPOGRAPHIC DATA USE FOR FLOOD EVENT MODELING OVER URBAN AREAS: TOWARD A SENSITIVITY ANALYSIS APPROACH
}

\author{
Morgan Abily ${ }^{1}$, Olivier Delestre ${ }^{2}$, Laura Amossé ${ }^{3}$, Nathalie Bertrand ${ }^{4}$, Yann \\ Richet $^{5}$, Claire-Marie Duluc ${ }^{6}$, Philippe Gourbesville ${ }^{7}$ and Pierre Navaro ${ }^{8}$
}

\begin{abstract}
D Free-surface hydraulic modeling tools are commonly used to assess flood hazard for production of maximal water depth $\left(h_{\max }\right)$ maps, as support for flood risk assessment. High Resolution (HR) topographic data are big data getting commonly available and used by hydraulic modeling community. Topographical information and its strategy of inclusion in models, are inputs of great importance for overland flow $h_{\max }$ calculation. To strengthen the assessment of confidence level in these deterministic hydraulic models outputs, uncertainty analysis (UA) and global sensitivity analysis (SA) can provide useful information that is required by practitioners and decision makers. UA and SA approaches allow to identify effective strategies to reduce the uncertainty of a model output. In this paper, developed approach consists in parameterizing three factors which introduce uncertainty related to HR topographic data use with hydraulic models: the measurement error (var. $E$ ), the level of details of above ground element representation in DEM (buildings, sidewalks, etc.) (var. $S$ ), and the spatial discretization resolution (grid cell size of a regular mesh) (var. $R$ ). Parameter var. $E$ follows a probability density function whereas parameter var. $S$ and var. $R$ are discrete operator choices. The coupling of an environment for parametric computation (Prométhée) and a code relying on 2D shallow water equation (FullSWOF_2D), Promethée-FullSWOF_2D (P-FS) tool has been set up. P-FS tool allows launching directly numerous set of computation using $R$ software. 1200 simulations of a river flood event scenario were performed on the regular computational mesh, spatially discretizing a 17.5 $\mathrm{km}^{2}$ urban area (Nice, France). The aim is to produce UA over points of interests and SA through Sobol index maps production.
\end{abstract}

\section{INTRODUCTION}

Over urban and industrialized areas, flood events might result in severe human, economic and environmental consequences. In mega-cities flood resilience context [Djordjević et al., 2011], as well as in a nuclear plant

1 Institut de Radioprotection et de Sûreté Nucléaire \& Lab. I-CiTy URE 005, EPU Nice Sophia, University of Nice, France, e-mail : abily@polytech.unice.fr, corresponding author

${ }^{2}$ Lab. J.A. Dieudonné UMR 7351 CNRS \& EPU Nice Sophia, University of Nice, France, e-mail : delestre@math.unice.fr

${ }^{3}$ EPU Nice Sophia, University of Nice, France, e-mail : amoslau1@aquacloud.net

${ }^{4}$ Institut de Radioprotection et de Sûreté Nucléaire, France, e-mail : nathalie.bertrand@irsn.fr

${ }^{5}$ Institut de Radioprotection et de Sûreté Nucléaire, France, e-mail : claire-marie.duluc@irsn.fr

${ }^{6}$ Institut de Radioprotection et de Sûreté Nucléaire, France, e-mail : richet.yann@irsn.fr...

7 Lab. I-CiTy URE 005, EPU Nice Sophia, University of Nice, France, e-mail : philippe.gourbesville@unice.fr

8 IRMA, UMR 7501 CNRS, Unistra, France, e-mail : navaro@math.unistra.fr

(C) EDP Sciences, SMAI 2015 
hardened shell core safety context, accurate flood risk maps are required. These maps should provide fine information on maximal water depth $\left(h_{\max }\right)$ and maximal flow velocity $\left(V_{\max }\right)$ reached during a given flood event simulation. This high resolution information helps to make detailed estimations of flood impact on city functions (e.g. services: health, energy, communication, transport, etc.) [Batica et al., 2013] as well as to enhance nuclear safety by strengthening flood protection. These information estimated by deterministic models, will be inputs for flood mitigation strategies development.

For practical flood modeling applications over urban and industrial areas, standard deterministic free surface hydraulic modeling approaches most commonly rely either on (i) 2D Shallow Water Equations (SWEs) codes, (ii) simplified version of 2D SWE (e.g. diffusive wave approximation [Moussa and Bocquillon, 2000]) or (iii) multiple porosity shallow water approaches [Guinot, 2012]. These approaches are different in terms of mathematical description of flow behavior, computational cost and required dissimilar quantity and type of input data. In cities or at large suburb scales, these methods give overall similar results. Nevertheless, at smaller scales (street, compound or buildings scale) for a high resolution description of overland flow properties $\left(h_{\max }\right.$ and $\left.V_{\max }\right)$ reached during a flood event, codes based on 2D SWEs using fine description of the environment are required. Indeed, above ground surface features (buildings, walls, sidewalks, etc.) that influence overland flow path are densely present. These structures have a high level of diversity in urban and industrial areas and create a complex environment for overland flow. Category of numerical codes based on 2D SWEs use with high resolution topographic information provide a valuable approach which has been previously tested for runoff modeling scenario at industrial site scale [Abily et al., 2013a, Abily et al., 2013b].

In the context of fluvial flood events modeling over a large urban environment scale, 2D SWEs based modeling tools are intensively used in practical studies. Urban reconstruction relying on airborne topographic data gathering technologies such as imagery and Light Detection and Ranging (LiDAR) scans are intensively used by Geomatics communities [Musialski et al., 2013]. These technologies allow to produce Digital Elevation Models (DEM) with a high accuracy level [Lafarge et al., 2010,Lafarge and Mallet, 2011, Mastin et al., 2009]. Moreover, modern technologies, such as an Unmanned Aerial Vehicle (UVA) use, make high resolution LiDAR or imagery born data easily affordable in terms of time and financial cost [Remondino et al., 2011, Nex and Remondino, 2013]. Consequently, hydraulic numerical modeling community increasingly uses HR DEM information from airborne technologies to model urban flood [Tsubaki and Fujita, 2010]. Among HR topographic data, photogrammetry technology allows the production of 3D classified topographic data [Andres, 2012]. This type of data is useful for surface hydraulic modeling community as it provides classified information on complex environments. It gives the possibility to select useful informations for a DEM creation specifically adapted for flood modeling purposes [Abily et al., 2013b].

Even-though HR classified data is of high horizontal and vertical accuracy (in a range of a few centimeters), this data set is assorted of errors and uncertainties. Moreover, in order to optimize models creation and numerical computation, hydraulic modelers make choices regarding procedure for this type of dataset use. These sources of uncertainties might produce variability in hydraulic flood models output. Dealing with uncertainties in hydraulic models is a press-forward concern for both practitioners [Iooss, 2011] and new guidance [ASN, 2013]. Sources of uncertainties in hydraulic models come from a hypothesis in mathematical description of the natural phenomena, from input parameters of the model, from numerical aspects when solving the model. Input parameters are of prime interest for applied practitioners willing to decrease the uncertainties in their models results. Hydraulic models input parameters have hydrological, hydraulic, topographical and numerical nature. Identification, classification and impact quantification of sources of uncertainties, on a given model output, are a set of analysis steps which will enable to (i) analyze uncertainties behavior in a given modeling problem, (ii) elaborate methods for reducing uncertainties on a model output, and (iii) communicate on relevant uncertainties. Uncertainty Analysis (UA) and Sensitivity Analysis (SA) methods are useful tools as they allow robustness of model predictions to be checked and help to identify input parameters effects. UA consists in the propagation of uncertainty sources through the model, and then focuses on trying to quantify the resulting uncertainty on model output [Saint-Geours, 2012]. It allows robustness of model results to be checked. Various methods are then available to rank parameters regarding their impact on result variability (such as Sobol index). 
This process goes one step beyond UA and constitutes a global sensitivity analysis (SA). SA stands in our study for global sensitivity analysis methods (omitting screening and local analysis methods) see [Jacques, 2011]. In practice, such type of approach is of a great interest, but is still at an exploratory level in applied studies relying on 2D SWE codes. Indeed, SA approach implementation is challenging, as it requires specific tools and deals with important computational capacity.

The purpose of the study presented in this paper is to provide both, (i) a tool and a protocol, to allow adaptable and ready-to use SA for 2D hydraulic modeling applications and (ii) to tackle impact of uncertainties related to HR topographic data use, over the variance of $h_{\max }$ calculated by $2 \mathrm{D}$ SWEs codes. Two categories of uncertain parameters are considered in our approach:

- first category is inherent to HR topographic data internal errors (measurement errors);

- second category is due to operator choices for this data inclusion in hydraulic codes.

The study is performed over the lower part of the Var river valley using an estimated hydrogram of the 1994 flood event. HR classified topographic data have been made available for the study area, which is $17.5 \mathrm{~km}^{2}$, by Nice municipality. The HR topographic data are included in numerical models under the form of DEM which is directly used as a computational grid (structured mesh). Three uncertain parameters are studied: the measurement error (var. E), the level of detail of aboveground element representation in DEM (buildings, sidewalks, etc.) (var. $S$ ), and the spatial discretization resolution (grid cell size for regular mesh) (var. $R$ ). A process using a coupling of an environment for parametric computation (Promethée) and a code relying on 2D shallow water equations (FullSWOF_2D) is developed (P-FS tool) and implemented on a high performance computing structure (Marseille Mésocentre).
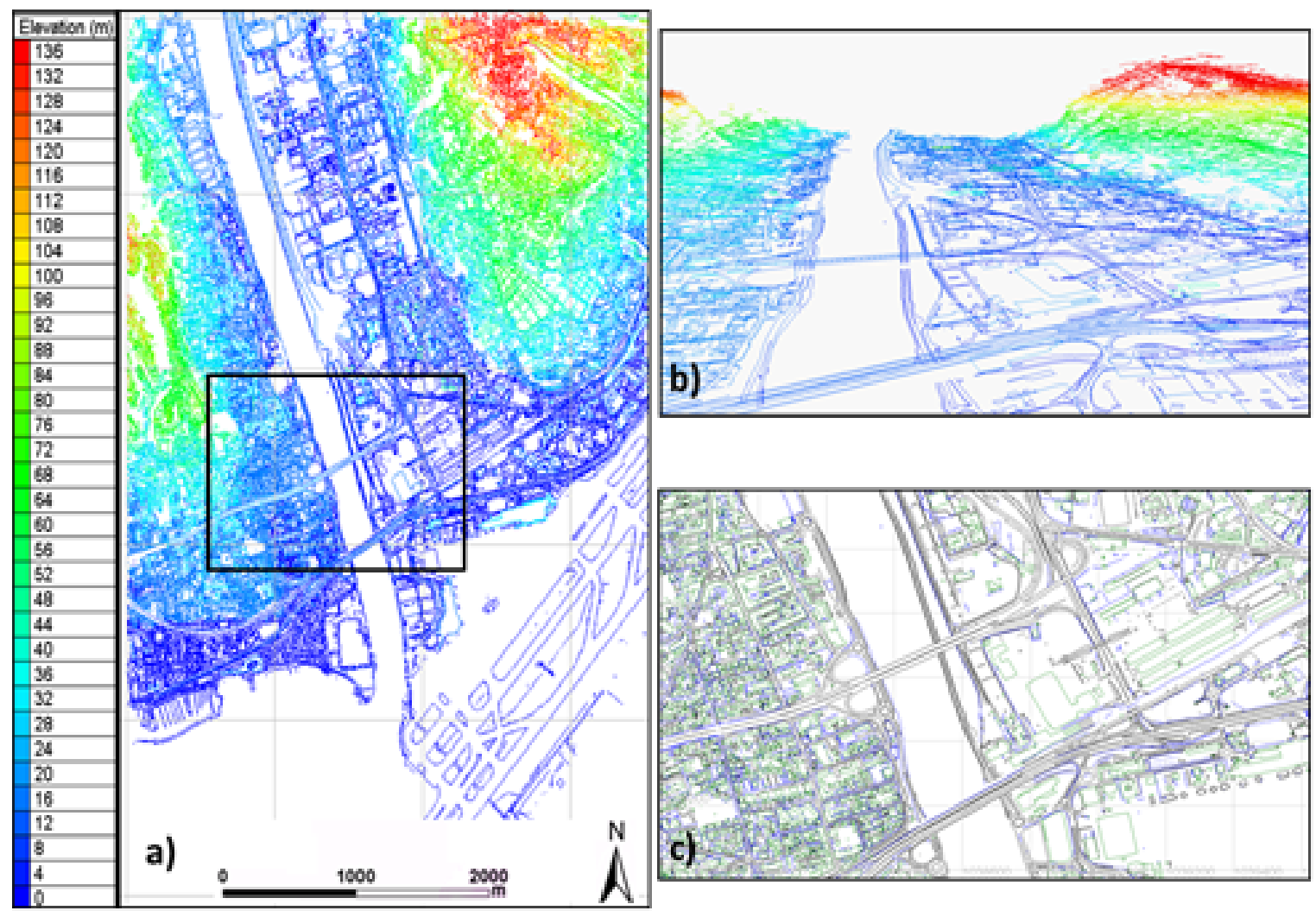

Figure 1. Overview (a) and zoom (b) of the HR 3D dataset selected classes with visualization (c) of buildings (green), walls (blue) and street concrete elements (black) 


\section{MAterial AND Methods}

\subsection{High resolution topographic data}

\subsubsection{Photogrammetric $3 D$ classified data : general aspects}

Aerial Photogrammetry technology allows to measure 3D coordinates of a space and its features using 2D pictures taken from different positions. The overlapping between pictures allows to calculate 3D properties of space and features based on stereoscopy principle. To measure accurately ground and features elevation, a step of aerotriangulation calculation is necessary, requiring information on picture properties regarding their position, orientation and bonding points. A low flight elevation, a high number of aerial pictures with different points of view and high levels of overlapping, allow to increase the accuracy and the reliability of the 3D coordinates measurement.

In photogrammetry, the spatial resolution is the size of a pixel at the ground level. For 3D classified data set creation a photo-interpretation step is necessary. Photo-interpretation allows creation of vectorial information based on photogrammetric dataset [Egels and Kasser, 2004, Linder, 2006]. A photo-interpreted dataset is composed of classes of points, polylines and polygons digitalized based on photogrammetric data. Important aspects in the photo-interpretation process are classes definition, vectorialisation methods and dataset quality used for photo-interpretation. These aspects will impact the design of the output classified dataset [Lu and Weng, 2007]. Class definition step has to be elaborated prior to the photo-interpretation step. The number, the nature and criteria for the definition of classes will depend on the objectives of the photo-interpretation campaign. Photo-interpretation techniques can be made (i) automatically by algorithm use, (ii) manually by a human operator on a Digital Photogrammetric Workstation (DPW) or (iii) by a combination of the two methods. The level of accuracy is higher when the photo-interpretation is done by a human operator on a DPW, but more resources are needed as the process becomes more time consuming [Lafarge et al., 2010]. Eventually, the 3D classification of features based on photo-interpretation allows to get 3D high resolution topographic data over territory which offer large and adaptable perspectives for its exploitation for different purposes [Andres, 2012].

Usually, when a photo-interpreted classified data set is provided to a user, the data is assorted with a global mean error value and with a percentage of photo-interpretation accuracy. The mean error value encompasses errors, due to material accuracy limits, to biased and to nuggets, which occurs within the photogrammetric data. The percentage of accuracy represents errors in photo-interpretation which can be feature misinterpretation, addition or omission.

\subsubsection{Low Var river Valley HR $3 D$ classified data}

A HR photogrammetric 3D classified data gathering campaign has been held in 2010-2011 over Nice municipality, covering $400 \mathrm{~km}^{2}$ [Andres, 2012]. The pixel resolution of aerial pictures is $0.1 \mathrm{~m}$ at the ground level. Features have been photo-interpreted under vectorial form in 50 different classes by human operators. These classes of elements include large above ground features such as building, roads, bridges, sidewalks, etc.. Thin above ground features, like concrete walls, road-gutters, stairs, etc., are included as well in classes. An important number of georeferencing markers are used (about 200). Globally, over the whole spatial extend of the data gathering campaign, the mean accuracy of the classified data is $0.3 \mathrm{~m}$ and $0.25 \mathrm{~m}$, respectively in horizontal and vertical dimension. Errors in photo-interpretation are estimated to represent $5 \%$ of the total number of elements. To control and ensure both, average level of accuracy and level of errors in photo-interpretation, the municipality has performed a terrestrial control of data accuracy over $10 \%$ of the domain covered by the photogrammetric campaign. For the low Var river valley area, a low flight elevation combined with a high level of overlapping among aerial pictures (80\%), have conduced to a higher accuracy level. In the low Var river valley sector, classified data horizontal and vertical mean accuracy is $0.2 \mathrm{~m}$. The total number of classified $3 \mathrm{D}$ polylines over this area is above 1,200,000. For our application, the 3D classified data of the low Var river valley is used to generate specific DEM adapted to surface hydraulic modeling. Therefore, only 3D classes of above ground feature, which are considered as impacting flow direction, are selected for DEM creation. It represents 12 classes, which includes buildings, concrete vertical structures above $2 \mathrm{~m}$ (walls) and low concrete features 
above paved roads (e.g. sidewalks, road gutter, etc.). These classes represent a total of 52,600 polylines over our area (figure 1). The 12 selected classes have been aggregated in 3 groups: buildings, "concrete" vertical structures (walls) and street concrete feature.

\subsection{Physically based model - FullSWOF_2D}

The name FullSWOF stands for Full Shallow Water equations for Overland Flow [Delestre et al., ]. It is a set of open source $\mathrm{C}++$ (ANSI) codes, freely available to the community (GPL- compatible license CeCILL-V2) from the website http://www.univ-orleans.fr/mapmo/soft/FullSWOF/. The structure of the code is made to facilitate the development of new evolutions. This software resolves the shallow water equations [Cunge et al., 1980] thanks to a well-balanced finite volume method based on the hydrostatic reconstruction (introduced in [Audusse et al., 2004,Bouchut, 2004]). This numerical method has good properties: water mass conservation, well-balancedness (at least preservation of a lake at rest equilibrium) and positivity water height preservation. Validations of FullSWOF_2D have already been performed on analytical solutions (SWASHES [Delestre et al., 2013]), on experimental data and on real events at small scales (agricultural parcels [Delestre et al., 2014]). The shallow water system in 2D (SW2D) writes:

$$
\left\{\begin{array}{l}
\partial_{t} h+\partial_{x}(h u)+\partial_{y}(h v)=0 \\
\partial_{t}(h u)+\partial_{x}\left(h u^{2}+g h^{2} / 2\right)+\partial_{y}(h u v)=g h\left(S_{0 x}-S_{f x}\right) \\
\partial_{t}(h v)+\partial_{x}(h u v)+\partial_{y}\left(h v^{2}+g h^{2} / 2\right)=g h\left(S_{0 y}-S_{f y}\right)
\end{array}\right.
$$

The first partial derivative equation of system (1) is the mass conservation equation and the two other ones are the momentum equation where the $2 \mathrm{D}$ vector $(u, v)$ is the fluid's horizontal average velocity across a vertical column of height $h(x, y, t)[m]$ and $g=9.81$ is the acceleration due to the gravity. The subscript $x$ (respectively $y$ ) stands for the x-direction (resp. the y-direction): $-S_{0 x}=\partial_{x} z(x, y)$ and $-S_{0 y}=\partial_{y} z(x, y)$ are the ground slopes and $S_{f x}$ and $S_{f y}$ the friction terms.

In FullSWOF, we have chosen to solve the SW2D on a structured grid. So we have chosen a numerical method adapted to the shallow water system in 1D (SW1D) or the Saint-Venant system [de Saint Venant, 1871] and then it is generalized to $2 \mathrm{D}$ thanks to the method of lines. So in what follows, we describe the numerical method for the SW1D. The SW1D writes

$$
\left\{\begin{array}{l}
\partial_{t} h+\partial_{x}(h u)=0 \\
\partial_{t}(h u)+\partial_{x}\left(h u^{2}+g h^{2} / 2\right)=g h\left(S_{0}-S_{f}\right)
\end{array}\right.
$$

in what follows, we consider Manning's friction law

$$
S_{f}=n^{2} \frac{u|u|}{h^{4 / 3}}=n^{2} \frac{q|q|}{h^{10 / 3}},
$$

with $q=h u$ the unit discharge $\left[\mathrm{m}^{2} / \mathrm{s}\right]$. The hydrostatic reconstruction is based on a general principle of reconstruction. We begin with a first order finite volume scheme for the form of SW1D (without source terms): choosing a positive and consistent numerical flux $\mathbf{F}\left(U_{L}, U_{R}\right)$ (e.g. Rusanov, HLL, kinetic, etc.), a finite volume scheme writes under the general form

$$
\frac{U_{i}^{*}-U_{i}^{n}}{\Delta t}+\frac{\mathbf{F}\left(U_{i}, U_{i+1}\right)-\mathbf{F}\left(U_{i-1}, U_{i}\right)}{\Delta x}=\overrightarrow{0}
$$

where $\Delta t$ is the time step and $\Delta x$ the space step. The idea is to modify this scheme by applying the flux to reconstructed variables. Reconstruction can be used to get higher order schemes (MUSCL, ENO, etc.), in that case higher order in time is obtained through TVD-Runge-Kutta methods [Shu and Osher, 1988]. And the aim of the hydrostatic reconstruction is to be well-balanced. It is designed to preserve at least steady states at rest $(u=0)$. Since [Bermúdez and Vázquez, 1994], it is well known that the topography needs a special treatment 
to preserve steady states without spurious oscillations. Schemes preserving at least lake at rest equilibrium are said to be well-balanced (a notion introduced in [Greenberg and LeRoux, 1996]). When it is directly applied to the initial scheme, it leads to an order one scheme, while coupling it with high order reconstruction increases the order and the accuracy of the scheme.

We describe now the implementation of this method for high order accuracy. The first step consists in performing a high order reconstruction (MUSCL, ENO, etc.). To properly treat the topography source term $\partial_{x} z$, this reconstruction is applied on $u, h$ and $h+z$, for more details see [Audusse and Bristeau, 2005]. This gives us the reconstructed variables $\left(U_{-}, z_{-}\right)$and $\left(U_{+}, z_{+}\right)$, on which the hydrostatic reconstruction is applied

$$
\left\{\begin{array}{l}
h_{i+1 / 2 L}=\max \left(h_{i+1 / 2-}+z_{i+1 / 2-}-\max \left(z_{i+1 / 2-}, z_{i+1 / 2+}\right), 0\right), \\
U_{i+1 / 2 L}=\left(h_{i+1 / 2 L}, h_{i+1 / 2 L} u_{i+1 / 2-}\right) \\
h_{i+1 / 2 R}=\max \left(h_{i+1 / 2+}+z_{i+1 / 2+}-\max \left(z_{i+1 / 2-}, z_{i+1 / 2+}\right), 0\right) \\
U_{i+1 / 2 R}=\left(h_{i+1 / 2 R}, h_{i+1 / 2 R} u_{i+1 / 2+}\right)
\end{array}\right.
$$

For a given space discretization, it may exhibit abnormal behaviors for some combinations of slope and water height [Delestre et al., 2012]. Particularly obvious for the order one scheme and on a coarse mesh, they disappear when refining the mesh, and are hardly noticeable at order two.

The finite volume scheme is modified as follows

$$
\frac{U_{i}^{*}-U_{i}^{n}}{\Delta t}+\frac{F_{i+1 / 2 L}^{n}-F_{i-1 / 2 R}^{n}-F c_{i}^{n}}{\Delta x}=\overrightarrow{0}
$$

where

$$
F_{i+1 / 2 L}^{n}=F_{i+1 / 2}^{n}+S_{i+1 / 2 L}^{n}, \quad F_{i-1 / 2 R}^{n}=F_{i-1 / 2}^{n}+S_{i-1 / 2 R}^{n}
$$

are left (resp. right) modifications of the numerical flux for the homogeneous system. In this formula, the flux is now applied to reconstructed variables $F_{i+1 / 2}^{n}=\mathbf{F}\left(U_{i+1 / 2 L}^{n}, U_{i+1 / 2 R}^{n}\right)$ and we take

$$
S_{i+1 / 2 L}^{n}=\left(\begin{array}{c}
0 \\
\frac{g}{2}\left(h_{i+1 / 2-}^{2}-h_{i+1 / 2 L}^{2}\right)
\end{array}\right), \quad S_{i-1 / 2 R}^{n}=\left(\begin{array}{c}
0 \\
\frac{g}{2}\left(h_{i-1 / 2+}^{2}-h_{i-1 / 2 R}^{2}\right)
\end{array}\right) .
$$

Finally, for consistency and well-balancing, a centered source term is added

$$
F c_{i}=\left(\begin{array}{c}
0 \\
-g \frac{h_{i-1 / 2+}+h_{i+1 / 2-}}{2}\left(z_{i+1 / 2-}-z_{i-1 / 2+}\right)
\end{array}\right) .
$$

The numerical strategy we choose consists in the HLL flux [Harten et al., 1983]

$$
\mathbf{F}\left(U_{L}, U_{R}\right)= \begin{cases}F\left(U_{L}\right) & \text { if } 0 \leq c_{1} \\ \frac{c_{2} F\left(U_{L}\right)-c_{1} F\left(U_{R}\right)}{c_{2}-c_{1}}+\frac{c_{1} c_{2}}{c_{2}-c_{1}}\left(U_{R}-U_{L}\right) & \text { if } c_{1}<0<c_{2} \\ F\left(U_{R}\right) & \text { if } c_{2} \leq 0\end{cases}
$$

with two parameters $c_{1}<c_{2}$ which are the approximations of the slowest and fastest wave speeds, respectively. We refer to [Batten et al., 1997] for further discussion on the wave speed estimates. The HLL flux is used with a modified MUSCL reconstruction [Bouchut, 2004]. It has shown to be the best compromise between accuracy, stability and CPU time cost (in [Delestre, 2010]). The MUSCL reconstruction [van Leer, 1979] of a scalar variable $s \in \mathbb{R}$ writes

$$
s_{i-1 / 2+}=s_{i}-\Delta x \cdot \frac{D s_{i}}{2}, \quad s_{i+1 / 2-}=s_{i}+\Delta x \cdot \frac{D s_{i}}{2},
$$


with the minmod slope limiter

$$
D s_{i}=\operatorname{minmod}\left(\frac{s_{i}-s_{i-1}}{\Delta x}, \frac{s_{i+1}-s_{i}}{\Delta x}\right), \quad \operatorname{minmod}(x, y)= \begin{cases}\min (x, y) & \text { if } x, y \geq 0 \\ \max (x, y) & \text { if } x, y \leq 0 \\ 0 & \text { else }\end{cases}
$$

In order to keep the discharge conservation, the reconstruction of the velocity has to be modified as

$$
u_{i-1 / 2+}=u_{i}-\frac{h_{i+1 / 2-}}{h_{i}} \frac{\Delta x}{2} D u_{i} \quad u_{i+1 / 2-}=u_{i}+\frac{h_{i-1 / 2+}}{h_{i}} \frac{\Delta x}{2} D u_{i}
$$

If we take $D s_{i}=0$, we recover the first order scheme in space. The friction term is taken into account by a fractional step, with the following system

$$
\partial_{t} U=\left(\begin{array}{c}
0 \\
-g h S_{f}
\end{array}\right)
$$

This system is solved thanks to a semi-implicit method (as in [Bristeau and Coussin, 2001])

$$
\left\{\begin{array}{l}
h^{n+1}=h^{*} \\
\frac{q^{n+1}-q^{*}}{\Delta t}=-n^{2} \frac{q^{n+1}\left|q^{n}\right|}{h^{n}\left(h^{n+1}\right)^{4 / 3}} .
\end{array}\right.
$$

This method allows to preserve stability (under a classical CFL condition) and steady states at rest. Finally a TVD-Runge Kutta method is applied to get the second order in time. For the generalization to 2D, we use the HLLC flux introduced in [Toro et al., 1994], combined with the method of lines. Concerning boundary conditions, we have modified the code, in order to have the discharge only in the riverbed, it is based on Riemann invariants. Finally, as we aim at simulating with big data, we have used a parallel version of FullSWOF based on a domain decomposition and the MPI library developped in the framework of CEMRACS 2012 [Cordier, S. et al., 2013]. This version has been compared and validated with an other parallel version based on SkelGIS library [Cordier, S. et al., 2013, Coullon et al., 2013].

\subsection{The low Var valley modeling scenario}

The 5th of November 1994, a flood event occurred in the Var catchment, leading to serious flooding in the low Var river valley [Guinot and Gourbesville, 2003]. In this paper, hydraulic conditions of this historical event will serve as a framework for a test scenario. The objective here is not to reproduce the flood event. Indeed, the site has changed since 1994: levees, dikes and urban structures have been intensively constructed in this area. As our approach aims at studying uncertainties related to HR topographic data use in hydraulic models, all the hydraulic parameters of the models are set identically for the simulations. Only the input DEM will change from one simulation to another following strategy defined in the next section. The flood scenario for our tests is based on an estimated hydrogram on the 5th of November 1994 event [Guinot and Gourbesville, 2003]. This hydrogram is our upstream boundary condition of the low Var river valley. To shorten the simulation length, we chose to simulate a 9 hours scenario (figure 2). First, a constant discharge of $1,500 \mathrm{~m}^{3} \cdot \mathrm{s}^{-1}$ is run for 3 hours to reach a steady state. This will serve as an initial condition for all the simulations. The overtopping part of the hydrogram is run, reaching the estimated peak discharge $\left(3,700 \mathrm{~m}^{3} \cdot \mathrm{s}^{-1}\right)$ and then decreasing long enough to observe a diminution of the overland flow water depth. The Manning's $n$ coefficient is spatially uniform on overland flow areas with a value of 0.015 which corresponds to a concrete surfacing [Chow, 1959]. No energy loss properties have been included in the hydraulic model to represent the bridges piers effects. Downstream boundary condition is an open sea level with a Neumann boundary condition. 


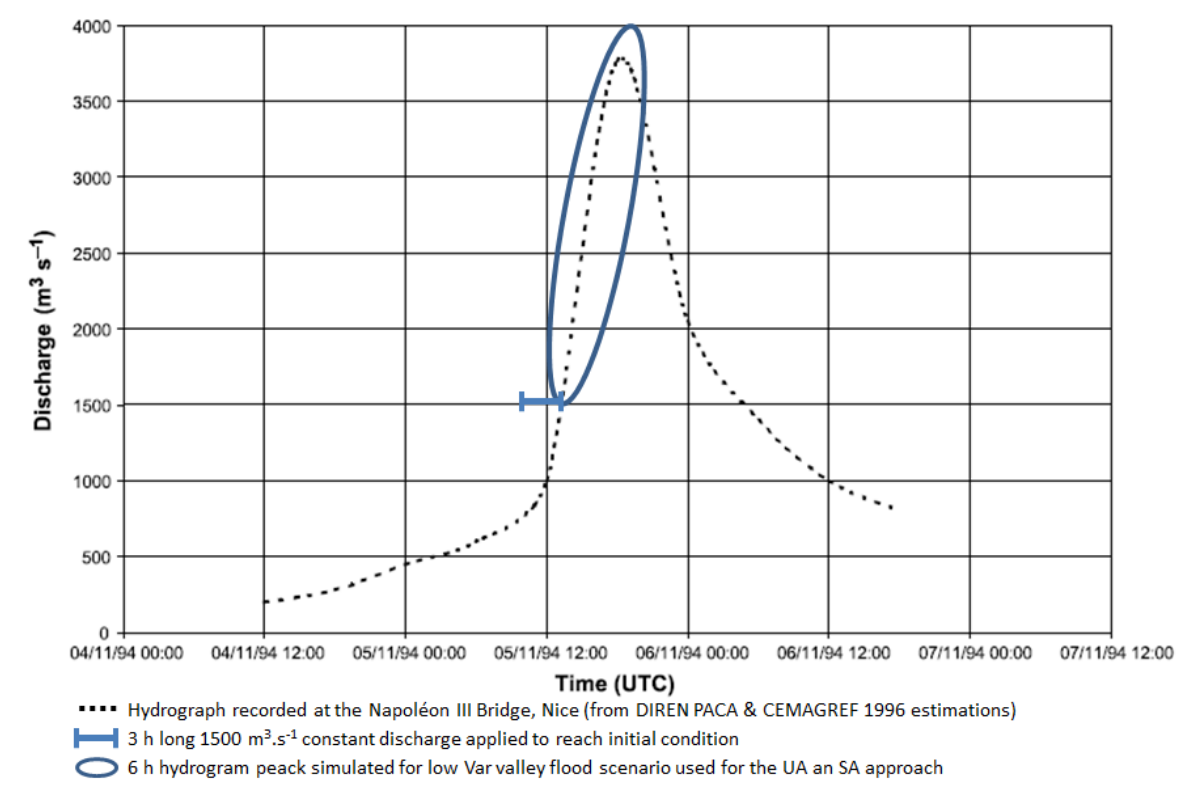

FiguRE 2. Estimated 1994 flood event hydrogram at the Napoléon bridge with schematization of simplification of the hydraulic scenario used for our UA orientated simulations.

\subsection{Material and method for the sensitivity analysis}

\subsubsection{Overview of the approach}

A schematization of our SA approach is presented in figure 3. As previously mentioned, this study focuses on two categories of parameters introducing uncertainty, in output of interest $h_{\max }$ calculation. These parameters are related to accuracy errors in HR classified data and to operator choices when building DEM and when integrating this DEM in hydraulic model. One parameter (var. E) encompasses uncertainty related to HR topographic data measurement errors. Two parameters (var. $S$ and var. $R$ ), represent uncertainty introduced by operator, respectively when building a specific DEM (var. $S$ ) and when spatially discretizing topographic information within 2D hydraulic code (var. $R$ ). Var. $E, S$ and $R$ properties are detailed in the next section. A sample of 2,000 DEMs which are used directly as structured mesh in the hydraulic code FullSWOF_2D is created. This sample of DEMs combines all the possibilities of selected sets of the three input parameters var. $S, R$ and $E$. Aim is to produce a database of variable output of interest $\left(h_{\max }\right)$ combining systematically parameters var. $S, R$ and $E$. This experience plan might not be optimal, but will allow to proceed to Monte Carlo sampling within the output database, verifying convergence of the Monte Carlo run, through the use of bootstrap test as a post-treatment phase. To run the 2,000 hydraulic simulations, Prométhée software is coupled with FullSWOF_2D. Prométhée is an environment for parametric computation allowing to carry the uncertainties propagation study when coupled to a code. This software is an open source environment developed by IRSN (http://promethee.irsn.org/doku.php). Interest of Prométhée lies in the fact that it will allow to parameterize input of any numerical code and is optimized for intensive computing resources use. Moreover, statistical post-treatment can be performed using Prométhée as it integrates $\mathrm{R}$ statistical environment. The coupled code Prométhée / FullSWOF (P-FS) is used to automatically launch parametrized computation.

For UA and SA, the deterministic code FullSWOF_2D is considered as a blackbox model as described in [Marrel et al., 2012]:

$$
\begin{aligned}
f: & \mathbb{R}^{p} \rightarrow \mathbb{R} \\
& X \mapsto Y=f(X),
\end{aligned}
$$



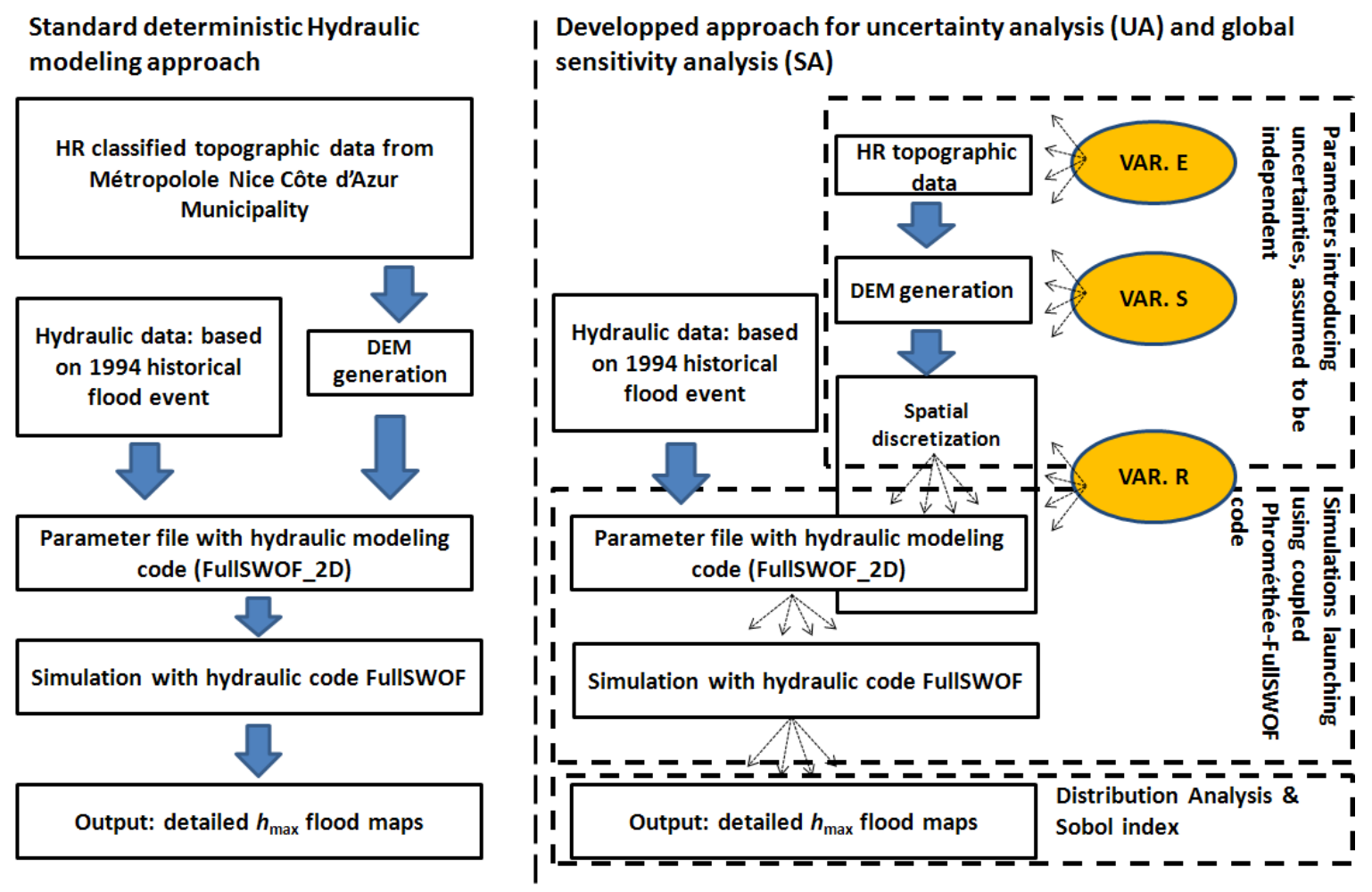

Figure 3. Schematisation of the experimental approach.

where $f$ is the model function, $X=\left(X_{1}, \ldots, X_{p}\right)$ are $p$ independent input random variables with known distribution and $Y$ is the output random variable. The UA will be done over the selected points of interest (figure 4). It is a first approach for analysing impacts of the three parameters over $h_{\max }$. Analysis is planned to be performed comparing the distribution at different points of $h_{\max }$ (minimum, maximum, mean, standard deviation and probability distribution shape). The principle of the SA method relies on estimation of the variance of the input variables (here $S, E$ and $R$ ) contribution to output variance (here $h_{\max }$ ). A unique functional analysis of variance (ANOVA) decomposition of any integrable function into a sum of elementary functions allows to define the sensitivity indices as explained in [Sobol', 1990, Marrel et al., 2012]. Sobol indices are defined as follow:

$$
S_{i}=\frac{\operatorname{Var}\left(\mathbb{E}\left(Y \mid X_{i}\right)\right)}{\operatorname{Var}(Y)} .
$$

For our approach, we plan to calculate first order Sobol indices for each of the 40 points of interest and then at each grid cell of the area of interest (figure 4).

\subsubsection{Application of the approach}

Parameters var. $S, E$ and $R$ are independent parameters considered as described below.

- Var. $S$ : modeler choices for DEM creation

It represents modeler choices for DEM creation. Four discrete stages are considered: (i) $S_{1}$, is the DTM of the study case, (ii) $S_{2}$, the elevation information of buildings added to $S_{1}$, (iii) $S_{3}$, the elevation information on the walls added to $S_{2}$, and (iv) $S_{4}$, elevation information of concrete features in streets added to $S_{3}$. Var. $S$ parameter is included in the SA as a categorical ordinal parameter. These discrete 


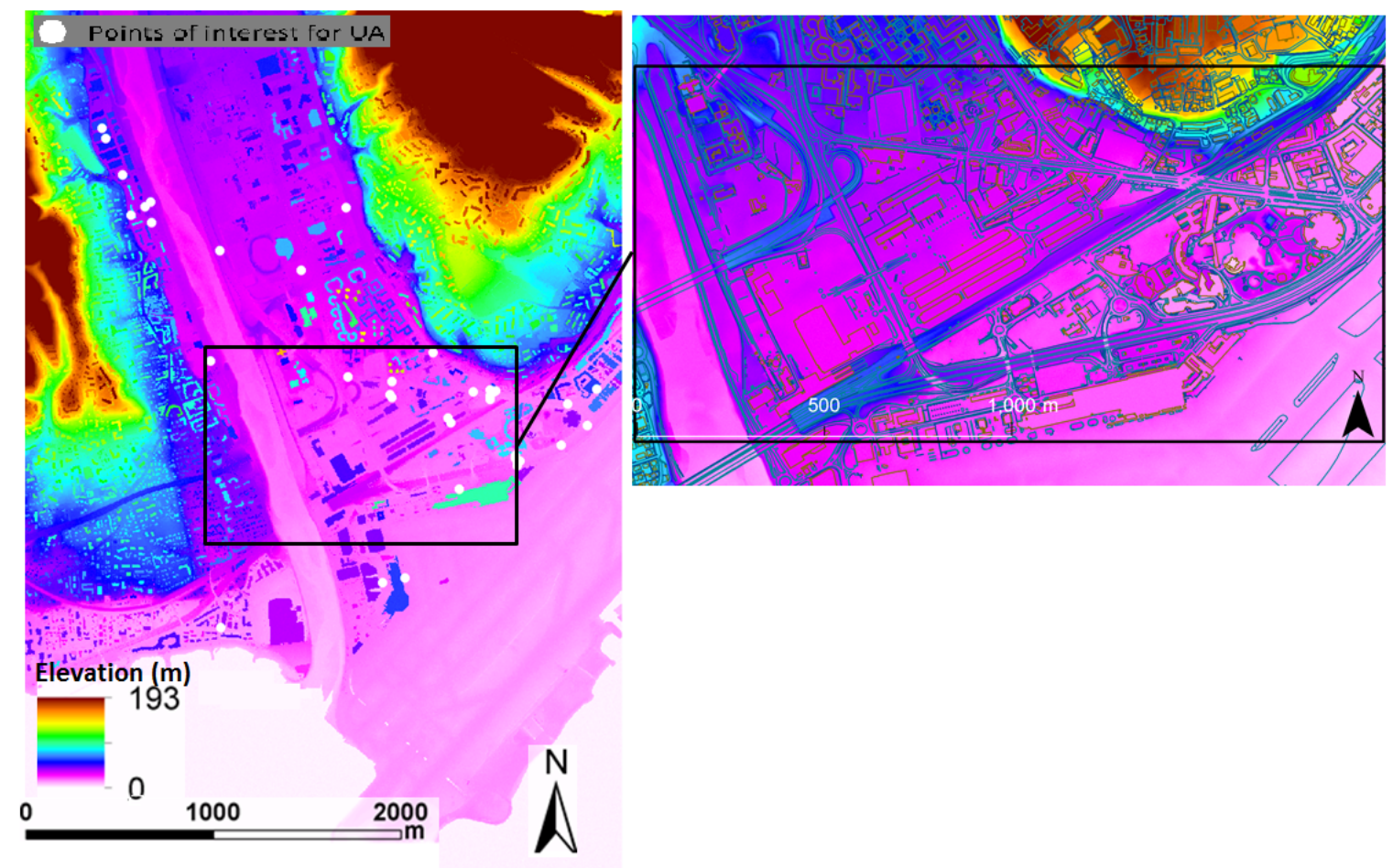

FIgURE 4. Location of points of interests for UA and focus on area of interest for Sobol index maps creation.

modeler choices are considered as having the same probability. Four DEM are generated at resolution $1 \mathrm{~m}, S_{1}$ to $S_{4}$.

- Var. E: measurement errors of HR topographic dataset

This parameter introduces in the DEM with the finest resolution $(1 \mathrm{~m})$ a random error in each cell of the DEM. For our study, only the altimetric errors are taken into account. Indeed, the planimetric dimension of the error is assumed to be relatively less significant for hydraulic study purpose compared to altimetric error. This altimetric measurement error follows a Gaussian probability density function $\mathcal{N}(0,0.2)$, where the standard deviation is equal to the mean global error value $(0.2 \mathrm{~m})$. This error introduction is spatially homogeneous. This approach is a first approximation: mean error could be spatialised in different sub-areas having physical properties which would impact spatial patterns of error value. Moreover, errors in photo-interpretation (classification) are not considered here. One hundred grids of random errors are generated, $E_{1}$ to $E_{100}$.

- Var. $R$ : modeler choices for mesh spatial resolution

When included in 2D models, HR DEM information is spatially and temporally discretized. FullSWOF is based on structured mesh, therefore the DEM grid can be directly included as a computational grid without effort for mesh creation. Nevertheless, for practical application, optimization of computational time/accuracy ratio often goes through a mesh degradation process when a HR DEM is used. Var. $R$ represents modeler choices when decreasing regular mesh resolution. Var. $R$ parameter can take 5 discrete values: $1,2,3,4$ or $5 \mathrm{~m}$. This range of possible discrete value for var. $\mathrm{R}$ has been selected on purpose to be compatible with modeler choices, when optimizing models, in regard with the range of different levels of details of above ground features (var. S). Indeed decreasing the finest resolution would lead to prohibitive computational time, whereas increasing the resolution above $5 \mathrm{~m}$ would make 
the use high resolution topographic information pointless. Therefore in practical engineering flood modelling applications, depending on available computer resources, all combinations of var. R / var. S are consistent.

DEM is a generic term for Digital Terrain Model (DTM) and for Digital Surface Model (DSM). If the DEM represents the elevation of the bare ground, then it is qualified as a DTM. If the DEM include information of above ground features elevation, it is called a DSM. In urban-like environments, when a DEM includes detailed elevation information with an infra-metric resolution, the DEM is qualified as a High Resolution (HR).

The use of classified data allow to include elevation information about thin above ground features (narrower than $1 \mathrm{~m}$ ) which is under the vectorial form, in DEM (grid form) at a one meter resolution as explained in following section. To create the HR DEMs, the following approach has been carried out. An HR DTM using multiple ground level information sources (points, polygons and polylines) is created and provided at a $0.5 \mathrm{~m}$ resolution by DIGNCA. The HR DEM resolution is here degraded to $1 \mathrm{~m}$ resolution. At this resolution the number of mesh cells is above 17.8 million. Then, a selection procedure among classified data is performed. This selection is achieved by considering concrete elements which can influence overland flow drainage path only. It includes dikes, buildings, walls and "concrete" above ground elements (such as sidewalks, road gutters, roundabout, doors steps, etc.). 12 classes are selected among the 50 classes of the 3D photo-interpreted dataset (figure 1). During this step, polylines giving information on elevated roads and bridges, which might block overland flow paths, are removed. The remaining total number of polylines is 52,600 . Selected above ground features are aggregated in 3 groups of features (buildings, walls and concrete street features). Extruding elevation information of selected polylines groups on the DTM $\left(S_{1}\right)$, four $1 \mathrm{~m}$ resolution DEMs, $S_{1}$ to $S_{4}$, are produced. The previously described method has allowed inclusion of thin elements impacting flow behavior of infra-metric dimension, oversized to metric size, in the $1 \mathrm{~m}$ resolution regular mesh. Then, 100 grids of var. $E$ are produced and added to var. $S_{1}, S_{2}, S_{3}$ and $S_{4}$ at resolution $1 \mathrm{~m}$. Eventually, these 400 DEMs are used to create 2,000 DEMs having a resolution ranging from 1 to $5 \mathrm{~m}$. DEMs are named $S_{m} R_{n} E_{x}$, with the parameters $m \in \llbracket 1,4 \rrbracket$, $n \in \llbracket 1,5 \rrbracket$ and $x \sim \mathcal{N}(0,0.2)$ used in P-FS.

\section{Results AND PERSPECTIVES}

Modifications on FullSWOF_2D code allow to run described river flood event scenario. A proof of concept of 3D HR classified data use for river flood modeling is given here (figure 5). Advantages of such an approach rely on (i) possibility to include detailed surface elements influencing overland flow, and in automatization and modularity of class selection for HR DSM production and, (ii) taking advantage of FullSWOF_2D numerical properties of mass conservation, well-balancedness and positivity preservation, which are relevant for HR overland flow modeling in urban areas.

Performed version of P-FS couple allows to run simulations with a selected set of input parameters (var. E, $S$ and $R$ ). Through $\mathrm{R}$ commands, it is possible to launch serial calculation. The coupled tool is operational on the HPC Mésocentre. P-FS would be transposable over any common HPC structures, requiring only slight changes in the coupling part of the codes. It is possible to run simultaneously up to 30 simulations.

For our simulations, calculations running times are important. Indeed, this computation time is CFL restriction dependent and therefore, is considerably affected by mesh resolution. Over a 12 cores node of the Mésocentre, the computation time is 2, 6, 12, 24, 40 hours respectively for 5, 4, 3, 2, $1 \mathrm{~m}$ resolution grids. Using about 200,000 CPU hours, it has been possible to run 1,200 simulations. The remaining 800 simulations are for $R_{2}$ and $R_{1}$ resolutions which are the most resource-demanding simulations. Due to the fact that these simulations are still missing, UA and consequently SA have not been fully achieved at this stage of the study.

Figure 6 illustrates perspectives for UA at a given point of interest. Output of interest $h_{\max }$ variability at this point is illustrated through maximal surface elevation use $h_{\max }+z$. For a given resolution (here $R_{3}$ ), a sample of 200 results is used. It consists of 4 random sub-sets of 50 simulation results among $S_{1} R_{3} E_{x}, S_{2} R_{3} E_{x}$, $S_{3} R_{3} E_{x}$ and $S_{4} R_{3} E_{x}$. Perspectives for further analysis are following. Once all of the 2,000 simulations will be run, a Monte Carlo sampling will be done. Through a resampling method use (bootstrap, see [Cohen and 


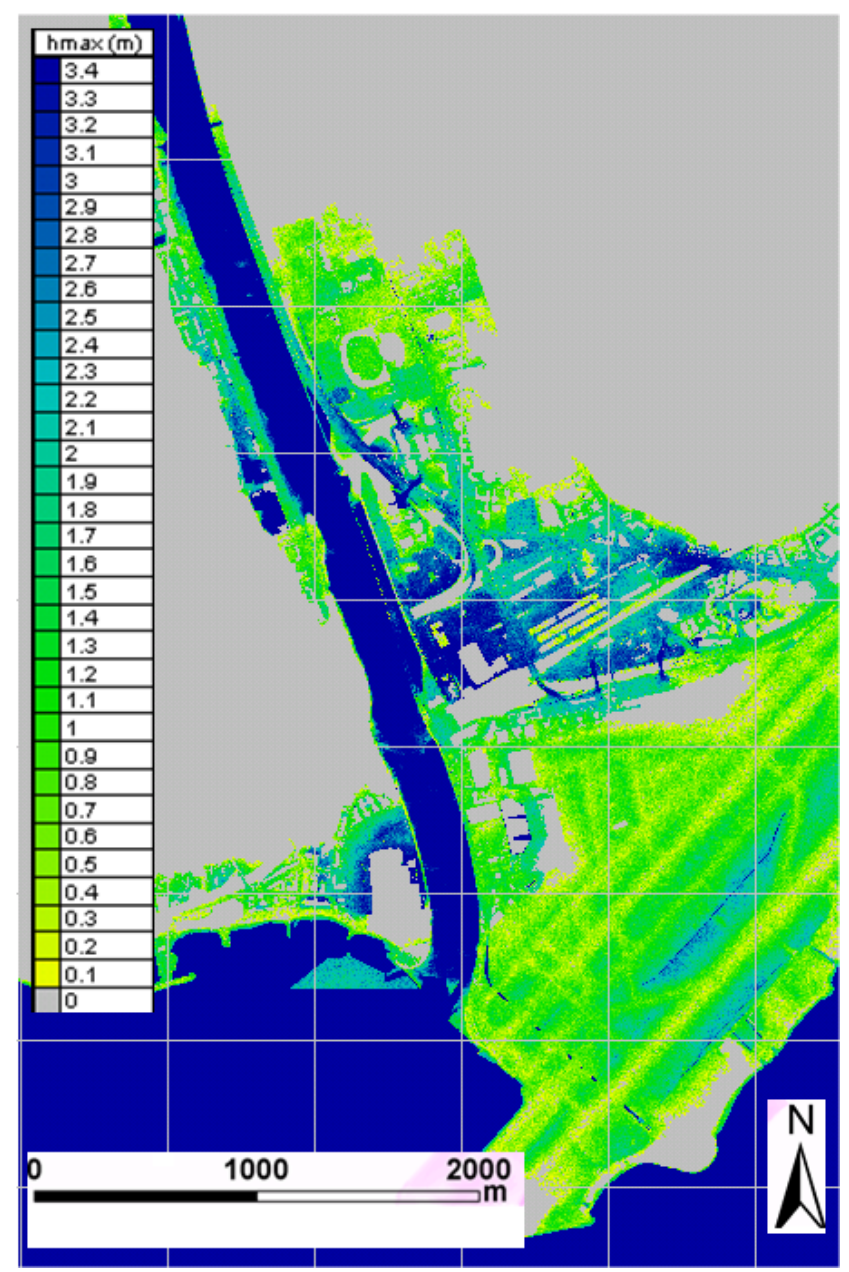

FIGURE 5. Illustration of $h_{\max }$ map obtained with HR topogrphic data use with P-FS for parameters $R_{3}, S_{4}, E_{4} 4$.

Cohen, 2008]), the convergence of the uncertainty propagation will be checked. Then analysis part of UA and SA will be possible.

Limits and possible improvements of our approach can be put to the light. For the finest resolutions $\left(R_{1}\right.$ and $R_{2}$ ), we might consider to increase the number of CPU used for computation. This will enable to reduce the running time of the simulations. The way measurement error (Var. E) has been taken into account is a first approximation. Indeed, it would be relevant to consider, in a more sophisticated approach, spatial zones where var. $E$ would have different PDF properties to better reproduce existing error spatial variability. This would require to put efforts in the characterization of errors measurements spatial variability. Moreover, errors related to photo-interpretation misinterpretation are not taken into consideration yet.

\section{ACKNOWLEDGMENTS}

This project has been founded by I-CiTy and IRSN. Photogrammetric and photo-interpreted dataset used for this study have been kindly provided by DIGNCA for research purpose. Technical expertise on Métropole Nice Côte d'Azur dataset has been provided by G. Tacet. This work was granted access to the HPC resources 


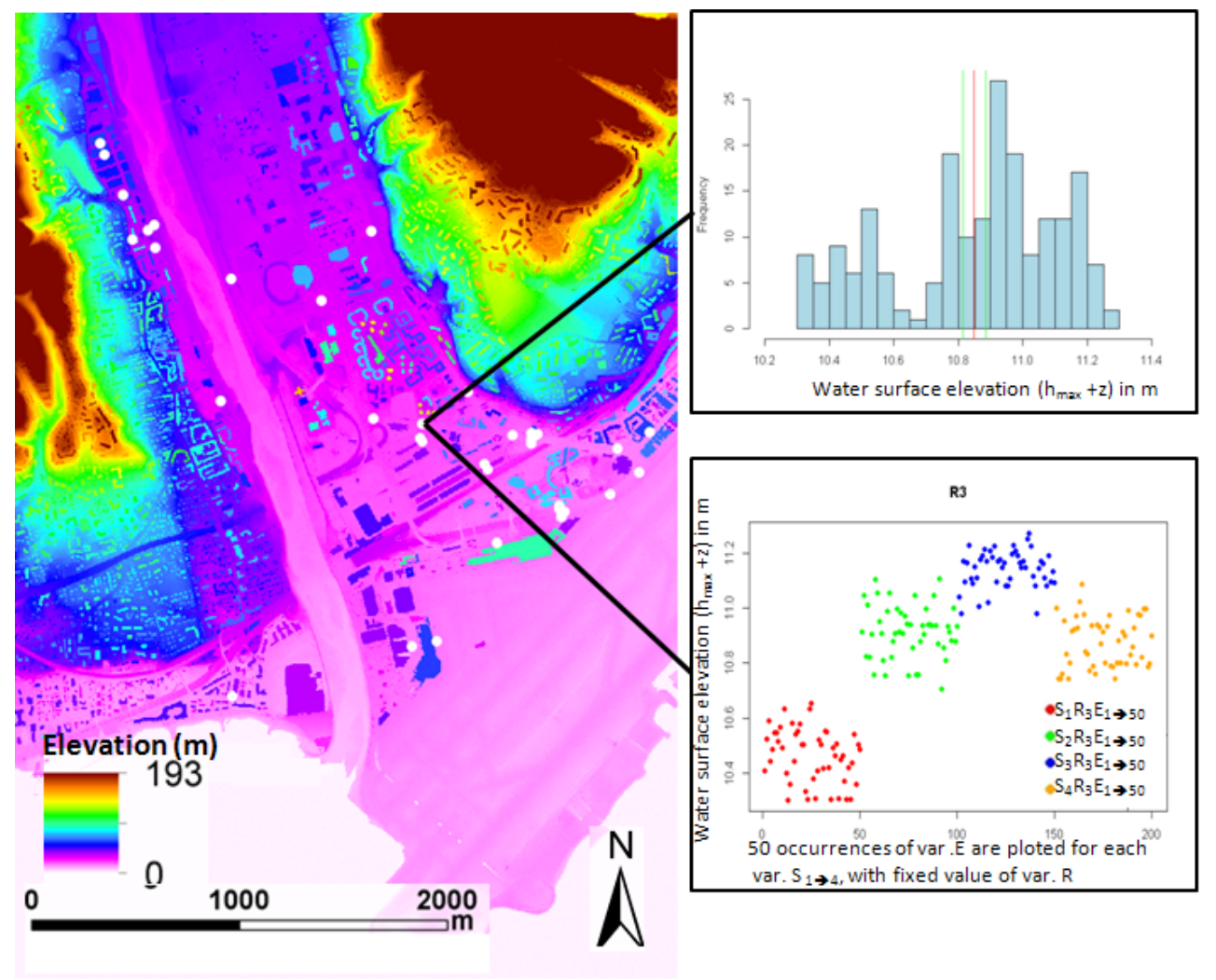

FIGURE 6. Illustration of UA possibilities at a point of interest using a sub-set of outputs.

of Aix-Marseille Université financed by the project equip@meso (ANR-10-EQPX-29-01) of the program "Investissements d'Avenir" sponsorised by the ANR. Technical support for codes adaptation on high performance computation centers has been provided by C. Laguerre, F. Lebas. and H. Coullon. Advices on uncertainty propagation have been kindly provided by B. Iooss, A. Marrel and CEMRACS organizers, in particular A. Nouy.

\section{REFERENCES}

[Abily et al., 2013a] Abily, M., Duluc, C. M., Faes, J. B., and Gourbesville, P. (2013a). Performance assessment of modelling tools for high resolution runoff simulation over an industrial site. Journal of Hydroinformatics, 15(4):1296-1311.

[Abily et al., 2013b] Abily, M., Gourbesville, Andres, L., and Duluc, C.-M. (2013b). Photogrammetric and LiDAR data for high resolution runoff modeling over industrial and urban sites. In Zhaoyin, W., Lee, J. H.-w., Jizhang, G., and Shuyou, C., editors, Proceedings of the 35th IAHR World Congress, September 8-13, 2013, Chengdu, China. Tsinghua University Press, Beijing.

[Andres, 2012] Andres, L. (2012). L'apport de la donnée topographique pour la modélisation 3d fine et classifiée d'un territoire. Revue XYZ, 133 - 4e trimestre:24-30.

[ASN, 2013] ASN (2013). Protection of Basic Nuclear Installations Against External Flooding - guide no.13 - version of 08/01/2013. Technical report, Autorité de Sûreté Nucléaire.

[Audusse et al., 2004] Audusse, E., Bouchut, F., Bristeau, M.-O., Klein, R., and Perthame, B. (2004). A fast and stable wellbalanced scheme with hydrostatic reconstruction for shallow water flows. SIAM J. Sci. Comput., 25(6):2050-2065. 
[Audusse and Bristeau, 2005] Audusse, E. and Bristeau, M.-O. (2005). A well-balanced positivity preserving "second-order" scheme for shallow water flows on unstructured meshes. Journal of Computational Physics, 206:311-333.

[Batica et al., 2013] Batica, J., Gourbesville, P., and Hu, F.-Y. (2013). Methodology for flood resilience index. In Butler, D., Chen, A. S., Djordjevic, S., and Hammond, M. J., editors, Proceedings of the International Conference on Flood Resilience: Experiences in Asia and Europe, held in Exeter, United Kingdom, 5-7 September 2013, page 302. Centre for Water Systems, University of Exeter.

[Batten et al., 1997] Batten, P., Clarke, N., Lambert, C., and Causon, D. M. (1997). On the Choice of Wavespeeds for the HLLC Riemann Solver. SIAM J. Sci. Comput., 18(6):1553-1570.

[Bermúdez and Vázquez, 1994] Bermúdez, A. and Vázquez, M. E. (1994). Upwind methods for hyperbolic conservation laws with source terms. Computers \& Fluids, 23(8):1049 - 1071.

[Bouchut, 2004] Bouchut, F. (2004). Nonlinear stability of finite volume methods for hyperbolic conservation laws, and well-balanced schemes for sources, volume 2/2004. Birkhäuser Basel.

[Bristeau and Coussin, 2001] Bristeau, M.-O. and Coussin, B. (2001). Boundary conditions for the shallow water equations solved by kinetic schemes. Technical Report 4282, INRIA.

[Chow, 1959] Chow, V. T. (1959). Open-Channel Hydraulics. McGraw-Hill.

[Cohen and Cohen, 2008] Cohen, Y. and Cohen, J. (2008). Statistics and Data with R: An Applied Approach Through Examples. Wiley.

[Cordier, S. et al., 2013] Cordier, S., Coullon, H., Delestre, O., Laguerre, C., Le, M. H., Pierre, D., and Sadaka, G. (2013). Fullswof paral: Comparison of two parallelization strategies (mpi and skelgis) on a software designed for hydrology applications. ESAIM: Proc., 43:59-79.

[Coullon et al., 2013] Coullon, H., Le, M.-H., and Limet, S. (2013). Parallelization of Shallow-water Equations with the Algorithmic Skeleton Library SkelGIS. Procedia Computer Science, 18(0):591-600. 2013 International Conference on Computational Science.

[Cunge et al., 1980] Cunge, J., Holly, F., and Verwey, A. (1980). Practical Aspects of Computational River Hydraulics. Pitman Publishing, London, T. Fisher Unwin.

[de Saint Venant, 1871] de Saint Venant, A. J.-C. (1871). Théorie du mouvement non-permanent des eaux, avec application aux crues des rivières et à l'introduction des marées dans leur lit. Comptes Rendus de l'Académie des Sciences, 73:147-154.

[Delestre, 2010] Delestre, O. (2010). Simulation du ruissellement d'eau de pluie sur des surfaces agricoles/ rain water overland flow on agricultural fields simulation. PhD thesis, Université d'Orléans (in French), available from TEL: tel.archivesouvertes.fr/INSMI/tel-00531377/fr.

[Delestre et al., 2014] Delestre, O., Cordier, S., Darboux, F., Du, M., James, F., Laguerre, C., Lucas, C., and Planchon, O. (2014). FullSWOF: A Software for Overland Flow Simulation. In Gourbesville, P., Cunge, J., and Caignaert, G., editors, Advances in Hydroinformatics, Springer Hydrogeology, pages 221-231. Springer Singapore.

[Delestre et al., 2012] Delestre, O., Cordier, S., Darboux, F., and James, F. (2012). A limitation of the hydrostatic reconstruction technique for Shallow Water equations/Une limitation de la reconstruction hydrostatique pour la résolution du système de SaintVenant. C. R. Acad. Sci. Paris, Ser. I, 350:677-681.

[Delestre et al., ] Delestre, O., Darboux, F., James, F., Lucas, C., Laguerre, C., and Cordier, S. FullSWOF: A free software for the simulation of shallow water flows. Submitted.

[Delestre et al., 2013] Delestre, O., Lucas, C., Ksinant, P.-A., Darboux, F., Laguerre, C., Vo, T.-N.-T., James, F., and Cordier, S. (2013). SWASHES: a compilation of shallow water analytic solutions for hydraulic and environmental studies. International Journal for Numerical Methods in Fluids, 72(3):269-300.

[Djordjević et al., 2011] Djordjević, S., Butler, D., Gourbesville, P., Mark, O., and Pasche, E. (2011). New policies to deal with climate change and other drivers impacting on resilience to flooding in urban areas: the CORFU approach. Environmental Science Es Policy, 14(7):864-873. Adapting to Climate Change: Reducing Water-related Risks in Europe.

[Egels and Kasser, 2004] Egels, Y. and Kasser, M. (2004). Digital Photogrammetry. Taylor \& Francis.

[Greenberg and LeRoux, 1996] Greenberg, J. M. and LeRoux, A.-Y. (1996). A well-balanced scheme for the numerical processing of source terms in hyperbolic equation. SIAM Journal on Numerical Analysis, 33:1-16.

[Guinot, 2012] Guinot, V. (2012). Multiple porosity shallow water models for macroscopic modelling of urban floods. Advances in Water Resources, 37(0):40-72.

[Guinot and Gourbesville, 2003] Guinot, V. and Gourbesville, P. (2003). Calibration of physically based models: back to basics? Journal of Hydroinformatics, 5(4):233-244.

[Harten et al., 1983] Harten, A., Lax, P. D., and van Leer, B. (1983). On upstream differencing and Godunov-type schemes for hyperbolic conservation laws. SIAM Review, 25(1):35-61.

[Iooss, 2011] Iooss, B. (2011). Revue sur l'analyse de sensibilité globale de modèles numériques. Journal de la Société Française de Statistique, 152(1):1-23.

[Jacques, 2011] Jacques, J. (2011). Pratique de l'analyse de sensibilité : comment évaluer l'impact des entrées aléatoires sur la sortie d'un modèle mathématique. http://math.univ-lille1.fr/ jacques.

[Lafarge et al., 2010] Lafarge, F., Descombes, X., Zerubia, J., and Pierrot Deseilligny, M. (2010). Structural approach for building reconstruction from a single DSM. Trans. on Pattern Analysis and Machine Intelligence, 32(1):135-147. 
[Lafarge and Mallet, 2011] Lafarge, F. and Mallet, C. (2011). Building large urban environments from unstructured point data. In Computer Vision (ICCV), 2011 IEEE International Conference on, volume 0, pages 1068-1075, Los Alamitos, CA, USA. IEEE Computer Society.

[Linder, 2006] Linder, W. (2006). Digital Photogrammetry: A Practical Course. Springer Verlag.

[Lu and Weng, 2007] Lu, D. and Weng, Q. (2007). A survey of image classification methods and techniques for improving classification performance. International Journal of Remote Sensing, 28(5):823-870.

[Marrel et al., 2012] Marrel, A., Iooss, B., Veiga, S., and Ribatet, M. (2012). Global sensitivity analysis of stochastic computer models with joint metamodels. Statistics and Computing, 22(3):833-847.

[Mastin et al., 2009] Mastin, A., Kepner, J., and Fisher, J. I. (2009). Automatic Registration of LIDAR and Optical Images of Urban Scenes. In Computer Vision and Pattern Recognition, 2009. CVPR 2009. IEEE Conference on, pages 2639-2646.

[Moussa and Bocquillon, 2000] Moussa, R. and Bocquillon, C. (2000). Approximation zones of the saint-venant equations for flood routing with overbank flow. Hydrology and Earth System Sciences, 4(2):251-261.

[Musialski et al., 2013] Musialski, P., Wonka, P., Aliaga, D. G., Wimmer, M., van Gool, L., and Purgathofer, W. (2013). A survey of urban reconstruction. Computer Graphics Forum, 32(6):146-177.

[Nex and Remondino, 2013] Nex, F. and Remondino, F. (2013). UAV for 3d mapping applications: a review. Applied Geomatics, pages $1-15$.

[Remondino et al., 2011] Remondino, F., Barazzetti, L., Nex, F., Scaioni, M., and Sarazzi, D. (2011). UAV photogrammetry for mapping and 3D modeling - Current status and future perspectives. In Archives of Photogrammetry, Remote Sensing and Spatial Information Sciences, volume 38(1/C22). ISPRS Conference UAV-g, Zurich, Switzerland.

[Saint-Geours, 2012] Saint-Geours, N. (2012). Analyse de sensibilité de modèles spatialisés - Application à l'analyse coût-bénéfice de projets de prévention des inondations. These, Université Montpellier II - Sciences et Techniques du Languedoc.

[Shu and Osher, 1988] Shu, C.-W. and Osher, S. (1988). Efficient implementation of essentially non-oscillatory shock-capturing schemes. Journal of Computational Physics, 77(2):439-471.

[Sobol', 1990] Sobol', I. M. (1990). On sensitivity estimation for nonlinear mathematical models. Matematicheskoe modelirvanie, 2(1):112-118 (in Russian), MMCE, 1(4) (1993) :407-414 (in English).

[Toro et al., 1994] Toro, E., Spruce, M., and Speares, W. (1994). Restoration of the contact surface in the HLL-Riemann solver. Shock Waves, 4:25-34.

[Tsubaki and Fujita, 2010] Tsubaki, R. and Fujita, I. (2010). Unstructured grid generation using LiDAR data for urban flood inundation modelling. Hydrological Processes, 24:1404-1420.

[van Leer, 1979] van Leer, B. (1979). Towards the ultimate conservative difference scheme. V. A second-order sequel to Godunov's method. Journal of Computational Physics, 32(1):101-136. 